\title{
Generalized Anxiety and Major Depressive Symptoms of General Public in South Korea during the Early COVID-19 Pandemic
}

\author{
Kye S. Kim ${ }^{1}$, Peter M. Kang ${ }^{1}$ and He Sook N. Kim ${ }^{2, *}$ \\ ${ }^{1}$ Department of Medicine, Beth Israel Deaconess Medical Center and Harvard Medical School, Boston, MA 02215, USA \\ ${ }^{2}$ Department of Child \& Family Studies, Yonsei University, Seoul, 03722, South Korea \\ ${ }^{*}$ Corresponding Author: He Sook N. Kim. Email: hesooknancy@gmail.com
}

Received: 08 March 2021 Accepted: 01 June 2021

\begin{abstract}
The extent of viral spread and strategies in dealing with the COVID-19 pandemic have been different in each country. There are overall increased mental health concerns in many countries but it is unclear what the general public individuals who do not have heighten vulnerability to stressors for existing mental diseases or significant physical illnesses were experiencing during the pandemic. We evaluated the stressors and mental health of general public in South Korea that has a relatively low confirmed cases and deaths. Responses on the on-line survey questions were used to assess the mental and physical symptoms in association with individuals' reported stressors. Individuals with the symptoms of the generalized anxiety disorder (GAD) were reported in $58.9 \%$, and major depression (MD) in 23.2\%, mostly in minimal to mild degrees, cut-off scores of 5 and 10, respectively by the validated screening tools, GAD-7 and PHQ-9. Both GAD and MD symptoms were in $21.5 \%$ of the respondents. The total number of stress had significant association with the scores of GAD-7, PHQ-9, physical symptoms, sleep difficulties and resilience $(p<0.01)$. GAD scores were also associated with sleep difficulties $(p<0.01)$ and raising young children $(p<0.05)$. MD scores were associated with sleep difficulties, job-dissatisfaction, and educational level $(p<0.05)$. The limitations of the study include small sample size, usage of smartphone or email, potential under-reporting by stigma in the socio-cultural context and evolving nature of pandemic. We conclude that keeping careful watch for mental symptoms, stressors, sleep difficulties and other physical symptoms are important even for the individuals without previous mental illnesses during the pandemic era.
\end{abstract}

\section{KEYWORDS}

Generalized anxiety disorder symptoms; major depression symptoms; sleep difficulties; physical symptoms; pandemic stressors; South Korea; generalized anxiety disorder-7(GAD-7); patient health questionnaire-9(PHQ-9)

\section{Introduction}

The COVID-19 crisis, declared as a pandemic on March 11, 2020 by the World Health Organization (WHO), has been threatening people with widely spread infection and deaths around the globe. As of May 8, 2021, the total global confirmed cases reported to WHO are well-over 156.4 million people and 3.2 million death. The cases have also been accumulating in South Korea but in a much smaller number, about 126,000 total confirmed and 1,860 death [1]. Among the Organization for Economic Co-operation and Development (OECD) nations reporting to WHO, after adjustment by the population size, 
South Korea was ranked $3^{\text {rd }}$ lowest in the number of confirmed cases and $2^{\text {nd }}$ lowest in the number of deaths in the year 2020 [1,2]. Since the first confirmed case on January $20^{\text {th }}, 2020$, South Korea has managed the crisis by implementing physical distancing and wearing mask in addition to innovative testing and exposuretracing without complete lock-down [3]. Although the challenges of subclinical manifestation of infection have been present since earlier time [4] and the clusters of group infection are still occurring [2], collaboration between the leaders implementing the guidelines timely manner and the general public willingly adhering to them have limited a wide spread of infection by both symptomatic and asymptomatic carriers [5]. Despite these well-coordinated and mitigating preventative strategies, examining self-perceived symptoms of South Korean general public may provide an understanding of mental health issues due to or brought out by the pandemic, aside from the impact of complete lockdown, inhomogeneous make-up of population (South Koreans are composed of $96 \%$ homogeneous ethnicity), politicized mask-wearing and other public health measures. With the opening of society occurring in many places, it might also provide an insight on the mental health during pandemic.

The infectious crisis due to Severe Acute Respiratory Syndrome (SARS) by SARS-CoV-1 virus had already shown to be associated with various mental disorders, such as increase of distress, depression, anxiety causing changes in sleep pattern, lethargy, and lack of hobby participation [6]. In the COVID-19 pandemic era, the extent of viral spread and death tolls are much more around the globe. Some may also have been experiencing symptoms but hesitant to seek medical help [7]. Significantly increased nonCOVID-19 mortality of patients who never made it to the hospital from New York City in USA and the Lombardi region in Italy were alarming [8]. Increased risk of death from other life-threatening, nonCOVID-19, medical emergencies in the states with large numbers of COVID-19 associated deaths were also warning us of the adverse, medical impacts [9]. For anxiety and depressive disorder symptoms, the Centers for Disease Control and Prevention (CDC) reported a substantial increase in the USA during April through June 2020 compared to that of the same time frame in 2019 [10]. For restricted access for non-COVID-19 care and public's hesitance to visit medical facilities, the increased extent of mental illnesses in pandemic remains short-sighted. In this study, we explored the mental health evaluation by surveying the South Korean general public during the pandemic by on-line responses of the self-reported mental and physical symptoms along with other associated variables. We focused on the individuals who do not already have diagnosis of mental or other significant physical illnesses, or other settings that heighten the responses to stressors. We intended to evaluate the frequency of common mental health symptoms that the general public was experiencing, without the influence of significant previous illnesses, treatments and or side effects. Non-diagnosed psychiatric or physical symptoms during the pandemic were assessed.

\section{Methods}

\subsection{On Line Survey}

We conducted an on-line survey from May 26 through August 04, 2020 in South Korea. Any adult older than 18 years old, with an access to a smart phone or internet who agreed to the disclosure and privacy statements was allowed to participate. Instead of using a specific internet shopping program or social media, "word or mouth" approach by cell phones and electronic mails were used for data gathering. Informed consents were obtained from all 303 respondents before starting the survey. Their responses were voluntary. As a token of appreciation for participating in the survey, a merchandise coupon worth $5,000 \mathrm{KW}(\sim \$ 4.50 \mathrm{USD})$ was sent to those who indicated interest.

The exclusion criteria included: (1) previously diagnosed mental illnesses, (2) significant physical illnesses, such as thyroid diseases, endocrinopathy, cancer or active chemotherapy or treatments, (3) RTPCR confirmed or history of COVID-19 infection, (4) recent bereavement within a year, (5) recent pregnancy or delivery within a year (6) recent divorce or domestic violence, and (7) incomplete responses 
to the survey. The additional exclusion criteria for the subgroup analyses of the parents bringing-up children were: (1) living with elderly parents or relatives who might be at high risk of mortality and morbidity if caught COVID-19, (2) single parent, and (3) raising more than one child.

\subsection{Survey Questions}

The survey was composed of 6 sections: (1) survey purposes, disclosures and consenting; (2) demographics; (3) mental and physical symptoms; (4) self-perceived health status, resilience and relationship with others; (5) illnesses and life style choices; and (6) SARS-Cov-2 related public health information, feed-back on the survey and respondent's interests in follow-up survey. The survey was written in Korean and estimated to be completed in about an hour.

The survey questions were based on the validated screening questions, such as the Generalized Anxiety Disorder-7 (GAD-7) and Patient Health Questionnaire-9 (PHQ-9) for Major Depression (MD) [11,12]. In this paper, GAD-7 symptom score is referred as the anxiety score and PHQ-9 symptom score is referred as the depression score. It must be noted, however, the symptoms of GAD-7 or PHQ-9 scores do not equate a diagnosis of GAD or MD which is also true for traumatic stress (TS) or post-traumatic stress disorder (PTSD). The questions of TS and PTSD symptoms were based on the Diagnostic and Statistical Manual of Mental Disorder-5 (DSM-5). Commonly experienced physical symptoms in medicine were also included for the respondents to answer in both open-ended and close-ended type of questions that included heart-racing sensations, dyspnea, headache, abdominal pain, sleep difficulties and others. In another question, the respondents were asked to select the symptoms that they were experiencing among the mental and physical symptoms listed. The questions regarding the sleep difficulties were about the sleep duration and quality as well as the interval changes compared to the time before the pandemic. To keep the survey in a reasonable length, not all of the items in the various instruments of insomnia evaluation were included [13,14]. Detailed methodology could be found in the Supplemental Methods.

We inquired about the stressors that each individual was experiencing by both open and close ended questions. We also inquired about the potential stresses experienced by the individuals that could arise from living with other co-inhabitants in a household. Individual's self-perceived, resilience in response to stress and one's stress management facet such as being able to carry out hobby during pandemic in the number of times per month were also asked. The questions about the self-perceived health status and any changes since the pandemic began, as well as whether the health was maintained or deteriorated during the pandemic were asked along with the reasons for such. Open-ended, overall feedback about the survey, if it was found helpful or not, and the reasons were asked. Finally, the interest in the follow-up survey was also examined.

\subsection{Analyses}

The responses were analyzed using the Statistical Package for the Social Sciences 25.0 (SPSS 25.0). The data were first analyzed by Pearson correlation. Spearman Correlation was used for the variables such as gender and age. For multivariate analysis, potential compounding factors, such as age, gender, anxiety scores for depression analysis and depression scores for anxiety analysis, living in the outbreak regions, were held constant. Calculation of positive responses of the participants and the number of individuals with the ranges of GAD and Depression symptom scores were assessed according to the validated GAD-7 and PHQ-9 scoring system. Statistical significance was considered $p<0.05$.

\section{Results}

\subsection{Demographics and Characteristics of Respondents}

The total number of respondents was 246 after applying the exclusion criteria. The mean age was 40.3 years old. Only two individuals were 70 years old or older. Women and men were $66.7 \%$ and 
$33.3 \%$, respectively. Majority of respondents, $85.0 \%$, were living with family or friends and $63.4 \%$ were married. Twelve individuals out of 246 reported having had SARS-Cov2 RT-PCR testing that were all negative. All 246 individuals had no history of COVID-19 infection. The details of demographics and characteristics of our survey respondents are summarized in the Tab. 1. After excluding individuals living with their own parents, raising more than one child, and being single parent, the parents who were bringing-up a young child (less than the school-age) were 13.4\%, composed of 23 mothers and 10 fathers responded. The parents raising an older child were $11.0 \%$, composed of 14 mothers and 13 fathers responded. Other married individuals living with spouse without child or their own parents were 20.3\%, composed of 34 women and 16 men. To show the data representing most of regions of South Korea, the regions where individuals resided were also listed. Of note, our data of $41.9 \%$ were under-representing that region as Seoul, where $50 \%$ of population lives.

Table 1: Demographics and characteristics of respondents

\begin{tabular}{|c|c|}
\hline Items & Numbers $n=246(\%)$ \\
\hline $\begin{array}{l}\text { Age (years) } \\
<29 \\
30-39 \\
40-49 \\
50-59 \\
60-69 \\
>70\end{array}$ & $\begin{array}{l}47(19.1) \\
70(28.5) \\
72(29.3) \\
31(12.6) \\
24(9.8) \\
2(0.8)\end{array}$ \\
\hline $\begin{array}{l}\text { Gender: } \\
\text { Male } \\
\text { Female }\end{array}$ & $\begin{array}{l}82(33.3) \\
164(66.7)\end{array}$ \\
\hline $\begin{array}{l}\text { Marriage Status: } \\
\text { Married } \\
\text { Non-Married }\end{array}$ & $\begin{array}{l}156(63.4) \\
90(36.6)\end{array}$ \\
\hline $\begin{array}{l}\text { Last Completed Formal Education: } \\
\text { College and Beyond } \\
\text { High School }\end{array}$ & $\begin{array}{l}230(93.5) \\
16(6.5)\end{array}$ \\
\hline Employment & $164(66.7)$ \\
\hline Expecting Income Loss c/t Year 2019 & $139(56.5)$ \\
\hline Working in Health Care & $11(4.5)$ \\
\hline $\begin{array}{l}\text { Living in the Special City, Seoul } \\
\text { Living in Gyeonggi, Inchon region } \\
\text { Living in Metropolitan Cities } \\
\text { Living in Rural Regions } \\
\text { Living in the Major Outbreak Regions } \\
\text { Living Alone } \\
\text { Living with Family, Relatives or Friends } \\
\text { Parental Status: }\end{array}$ & $\begin{array}{l}103(41.9) \\
55(22.4) \\
20(8.1) \\
53(21.5) \\
15(6.1) \\
37(15) \\
209(85)\end{array}$ \\
\hline $\begin{array}{l}\text { Any Children* } \\
\text { Raising a Young Child** }\end{array}$ & $\begin{array}{l}115(46.7) \\
33(13.4)\end{array}$ \\
\hline
\end{tabular}




\begin{tabular}{ll}
\hline \multicolumn{1}{c}{ Table 1 (continued). } \\
\hline Items & Numbers $\mathrm{n}=246(\%)$ \\
\hline \multicolumn{1}{c}{ Raising an Older Child** } & $27(11.0)$ \\
Married and No Child** & $50(20.3)$ \\
Screen Hours/Day & \\
$<1 \mathrm{~h}$ & $180(73.2)$ \\
$1-2 \mathrm{~h}$ & $52(21.1)$ \\
$3-4 \mathrm{~h}$ & $12(4.9)$ \\
$>5 \mathrm{~h}$ & $2(0.8)$ \\
Hobby at least Once per Month & $215(87.4)$ \\
Sleep Difficulties in Duration and or Quality & $192(78.0)$ \\
\hline $\begin{array}{l}\text { Note: } \\
\text { Grandparents raising children regardless of age or number of children, living w/o their parents (a.k.a. }\end{array}$ \\
spouse, without child, and living w/o their parents (a.k.a. Grandparents) or relatives.
\end{tabular}

\subsection{Stressors and Symptoms Experienced during Pandemic}

Top 7 stressors and mental/physical symptoms that the respondents experienced during the pandemic were shown in Tab. 2. The respondents indicated their major stressors in pandemic involved the worries of getting infected from and infecting others and financial pressure. Interestingly, the concern for children's education and uncertain future caused by the pandemic were among the top stressors. As for the top frequently experienced mental and physical symptoms were decrease concentration or motivation, frequent tiredness, nervousness, headache, indigestion, heart-racing sensation and difficulty breathing in decreasing order. Mental symptoms were the first three most frequently experienced symptoms which is to be further explained in the latter part of this paper.

Table 2: Top Seven Stressors and Mental/Physical Symptoms

\begin{tabular}{|c|c|c|c|c|}
\hline \multicolumn{5}{|c|}{ Top Seven by the Number of Responses $(n=246)$} \\
\hline \multicolumn{2}{|c|}{ Rank Stressors in Pandemic } & \multicolumn{3}{|c|}{ Mental and Physical Symptoms } \\
\hline & Items & $\mathrm{N}(\%)$ & Items & $\mathrm{N}(\%)$ \\
\hline 1 & Getting Infected from Other people & $214(87.0)$ & $\begin{array}{l}\text { Decreased Concentration or } \\
\text { Motivation }\end{array}$ & $\begin{array}{l}120 \\
(48.8)\end{array}$ \\
\hline 2 & Infecting Others & $157(63.8)$ & $\begin{array}{l}\text { Frequent Tiredness without } \\
\text { Reason }\end{array}$ & $\begin{array}{l}110 \\
(44.7)\end{array}$ \\
\hline 3 & Getting Infected from Soiled Surfaces & $153(62.2)$ & Nervousness & $\begin{array}{l}99 \\
(40.2)\end{array}$ \\
\hline 4 & $\begin{array}{l}\text { Financial Pressure due to Economic } \\
\text { Down turn }\end{array}$ & $124(50.4)$ & Headache & $\begin{array}{l}74 \\
(30.1)\end{array}$ \\
\hline 5 & Loosening Health & $110(44.7)$ & Indigestion & $\begin{array}{l}73 \\
(29.7)\end{array}$ \\
\hline 6 & Children's Education/Uncertain Future & $68(27.6)$ & Heart Racing Sensation & $\begin{array}{l}52 \\
(21.1)\end{array}$ \\
\hline 7 & Getting or Maintaining Job & $51(20.7)$ & Dyspnea & $\begin{array}{l}42 \\
(17.1)\end{array}$ \\
\hline
\end{tabular}

Note: Multiple responses were possible for the questions inquiring about the stressors and mental/physical symptoms. $\mathrm{N}=$ number of individuals. 
The total number of stresses had significant associations with the scores of anxiety symptoms $(r=0.35$, $p<0.01)$, depression symptoms $(r=0.27, p<0.01)$, physical symptoms $(r=0.25, p<0.01)$, sleep difficulties $(r=0.24, p<0.01)$ and resilience $(\mathrm{r}=-0.23, p<0.01)$. Keeping-up with household payments or decreased income in 2020 were also a significant stress which was reported in a majority. Income loss due to pandemic was reported in $56.5 \%$ of respondents.

\subsection{GAD Symptoms}

About 58.9\% percent of respondents reported symptoms of GAD-7 in minimal ( $\mathrm{n}=105,42.7 \%)$, mild $(\mathrm{n}=37,15.0 \%)$ and moderate $(\mathrm{n}=3,1.2 \%)$ degree, with the mean $(M)$ anxiety score of $2.2,6.4$, and 11.7 , respectively (Fig. 1A). None was in higher degrees. The lowest mean anxiety and depression scores were in the age group 60-69 years old that were composed of retired individuals. The mean anxiety score in each age group was depicted in the Fig. 1B. Fifty percent of male respondents had anxiety symptoms $(M=1.5+/-0.2)$ and $42 \%$ of female respondents had the symptoms $(M=2.6+/-0.2)$.

A

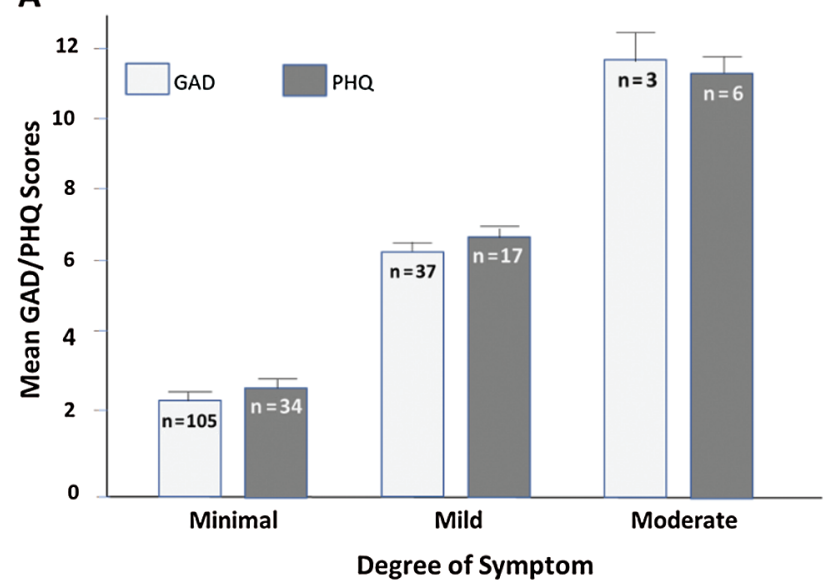

B

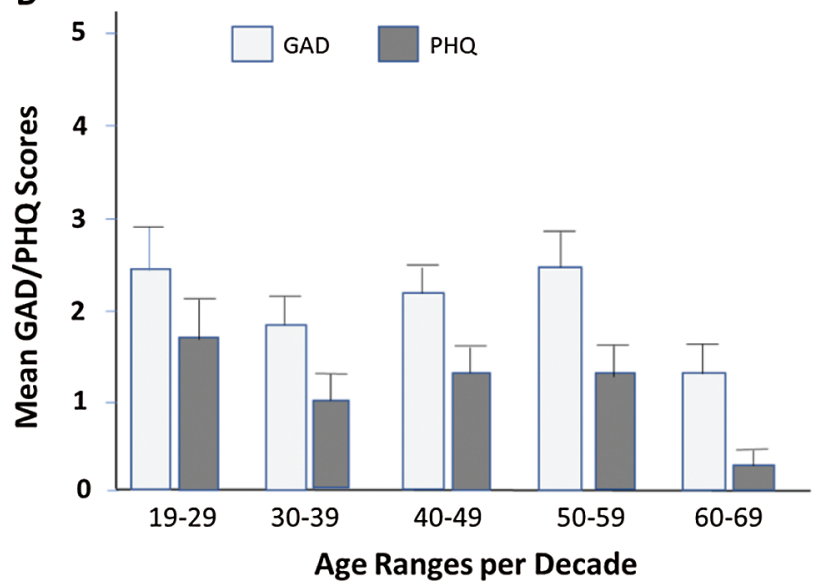

Figure 1: Mean Scores of Anxiety (GAD-7) and Depression (PHQ-9) Symptoms. A. Mean Scores of GAD-7 and PHQ-9 symptom by the severity. $\mathrm{n}=$ number of individuals in each group. B. Mean Scores of GAD-7 and PHQ-9 symptoms by the age ranges per decade

In addition, the anxiety scores were significantly associated with other variables $(p<0.01)$, such as the scores of PHQ-9 $(r=0.51)$ and sleep difficulties $(r=0.45)$ and physical symptoms $(r=0.25)$. The covariate effect analysis of the anxiety scores with the individual's self-perceived resilience, with and without the total number of stresses showed an interesting finding. The resilience alone did not have a significant association with the anxiety scores. However, when the total number of stresses was analyzed together, there was a significant association $(p<0.01)$. Anxiety scores also showed a significant inverse association with the number of times per month individuals carried out hobbies $(r=-0.18, p<0.01)$.

In the subgroup analyses, the parents bringing-up young child showed significant association with anxiety scores $(p<0.05)$, but not in the parents raising older child $(p>0.05)$. In these subgroups, the women's mean anxiety scores were considerably higher than the men's anxiety scores. The mean anxiety scores of mothers bringing-up young child $(M=3.6+/-0.8)$, fathers bringing-up young child $(M=1.8+/-0.6)$, mothers raising older child $(M=2.6+/-0.7)$ and fathers raising older child $(M=1.1+/-0.3)$. 


\subsection{Major Depression Symptoms}

Twenty-three percent of the respondents had depression symptoms at: minimal $(\mathrm{n}=34,13.8 \%)$, mild $(\mathrm{n}=17,6.9 \%)$ and moderate $(\mathrm{n}=6,2.4 \%)$ degrees with the mean score of 2.6, 7.1, and 11.3, respectively (Fig. 1A). None was in the moderately severe or severe degree which was the same for GAD. The Fig. 1B showed the mean depression score in each age group per decade. About $22 \%$ of the respondents had both symptoms of depression and anxiety.

Correlation and regression analyses done with the gender, age and other compounding variables held constant showed significant associations $(p<0.01)$ between depression scores and other variables, such as sleep difficulties $(r=0.49)$, total number of stresses $(r=0.27)$, job-dissatisfaction $(r=0.19)$, and educational back ground $(r=-0.18)$. Higher educational background was associated with less depressive symptom scores.

Unlike the significant association with anxiety scores $(p<0,05)$ shown in the parents bringing-up young child, no significant association with depression score was noted. Nevertheless, the mean depression scores of women were two times higher than those of men in both groups. The mean depression scores of mothers bringing-up young child $(M=2.1+/-0.1)$, fathers bringing-up young child $(M=0.4+/-0.1)$, mothers raising older child $(M=0.9+/-0.2)$ and fathers raising older child $(M=0.2+/-0.1)$.

\subsection{TS/PTSD Symptoms}

About $19 \%$ of individuals reported TS/PTSD symptoms. The individuals with TS/PTSD symptoms lasted more than one month were $6.4 \%$. Of those, $50 \%$ reported also having avoidance of the perceived culprit. The individuals who had the overlapping symptoms of TS/PTSD and anxiety, TS/PTSD and depression, and all three were, $13.8 \%, 9.3 \%$ and $8.1 \%$, respectively (Fig. 2). The individuals who had all three symptoms also reported financial melt-down, burn-out, lacking any hope for recovery, poverty, increased smoking and alcohol intake, health deterioration, job-related-stress, and/or Hwa Byung (a disorder or phenomenon related to accumulated stress without source of relief found in South Korean).

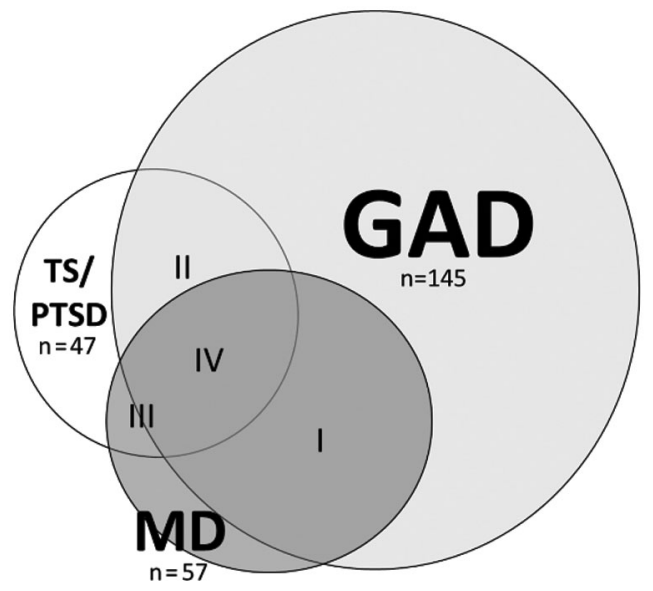

Figure 2: Overlap of GAD, TS/PTSD and MD Symptoms. Each circle represents the individuals with GAD, MD or TS/PTSD symptoms. Overlapping Area I = Individuals with GAD and MD symptoms $(\mathrm{n}=53$, 21.5\%), Overlapping Area II = Individuals with GAD and TS/PTSD symptoms $(\mathrm{n}=34,13.8 \%$ ), Overlapping Area III = Individuals with MD and TS/PTSD symptoms $(\mathrm{n}=23,9.3 \%)$, and Overlapping Area IV = Individuals with all 3 symptoms $(n=20,8.1 \%) . n=$ number of individuals in each group 


\subsection{Physical Symptoms}

One or more physical symptoms were reported in 98 individuals (39.8\%). Of note, sleep difficulties were not included among the physical or mental symptoms in our study, and analyzed separately because of the reciprocal relationship existed. The respondents reported more frequently of mental symptoms than physical symptoms when they were asked to select their symptoms among the list of common mental and physical symptoms (Tab. 2). The scores of physical symptoms had significant association with total number of stress, anxiety, depression, TS/PTSD, sleep difficulty and living alone $(p<0.05)$. Smell and/or taste loss were reported in those $(\mathrm{n}=7,3 \%)$ who had influenza, or sinus-nasal infections/inflammation, mostly with complete recovery (86\%). Only one individual of the survey respondents $(n=303)$ had COVID-19, confirmed by RT PCR positivity, who reported a complete recovery from the loss of smell and taste.

\subsection{Maintaining vs. Deteriorating Health (Tab. 3)}

The respondents' top reasons for maintaining health by self-perception were: keeping up with public health measures such as wearing masks, keeping physical distance, and washing hands appropriately $(60.2 \%)$, exercise and eating healthy $(29.7 \%)$, and having a new routine $(17.5 \%)$. In contrast, the top reasons for self-perceived, deteriorating health were: inability to exercise or carry out hobbies (40.2\%), inability to eat healthy $(29.7 \%)$, and lack of new routine $(25.2 \%)$. Of note, hesitance to seek medical help for fear of getting infected was also one of the top 7 reasons of deteriorating health $(11.8 \%)$. The stresses about children's education and uncertain future were also perceived reasons for their deteriorating health $(11.4 \%)$.

Table 3: Top Seven reasons for Maintaining Health vs. Deteriorating Health

\begin{tabular}{|c|c|c|c|c|}
\hline \multicolumn{5}{|c|}{ Top Seven Reasons $(\mathrm{n}=246)$} \\
\hline \multirow[t]{2}{*}{ Rank } & \multicolumn{2}{|c|}{ Maintaining Health during Pandemic } & \multicolumn{2}{|c|}{ Deteriorating Health during Pandemic } \\
\hline & Items & $\begin{array}{l}\text { Numbers } \\
(\%)\end{array}$ & Items & $\begin{array}{l}\text { Numbers } \\
(\%)\end{array}$ \\
\hline 1 & $\begin{array}{l}\text { Wearing mask, physical distancing and } \\
\text { washing hands appropriately }\end{array}$ & $\begin{array}{l}148 \\
(60.2)\end{array}$ & Inability to exercise or hobby & $99(40.2)$ \\
\hline 2 & Exercise and eating healthy & $73(29.7)$ & Inability to eat healthy & $73(29.7)$ \\
\hline 3 & Having a new routine & $43(17.5)$ & Lack of new routine & $62(25.2)$ \\
\hline 4 & Quitting/decreased smoking or drinking & $42(17.1)$ & Stressed about getting infected & $47(19.1)$ \\
\hline 5 & Clean air & $31(12.6)$ & Financial pressure & $38(15.4)$ \\
\hline 6 & $\begin{array}{l}\text { Time to rest due to shorter hours at work } \\
\text { or commute }\end{array}$ & $21(8.5)$ & $\begin{array}{l}\text { Deferring medical care due to } \\
\text { hesitance }\end{array}$ & $29(11.8)$ \\
\hline 7 & Being able to sleep well & $16(6.5)$ & $\begin{array}{l}\text { Stresses about the Children's } \\
\text { education \& uncertain future }\end{array}$ & $28(11.4)$ \\
\hline
\end{tabular}

Note: Multiple responses were possible for the questions inquiring the reasons of maintaining and deteriorating health during pandemic.

\subsection{Sleep Difficulties}

The sleep difficulty scores, assessing the interval changes compared to before the pandemic era in the sleep quantity and quality, showed significant association with the anxiety $(r=0.44)$, depression $(r=0.49$, TS/PTSD $(r=0.37)$, and physical symptoms $(r=0.46)$ (all items, $p<0.01)$ (Tab. 4). Sleep difficulty scores analyzed by regression was an explaining factor for anxiety scores $\left(F(1,243)=60.1 ; p<0.01 ; R^{2}=0.198\right)$. Of note, not all were included for this part of analysis for individuals' vague comments about sleep aid usage. 
Positive correlations in the sleep difficulty with the mean scores of anxiety and depression were also demonstrated (Fig. 3). The top reasons the respondents identified for their sleep difficulties were uncertainties about the future, difficulties in getting along with people, job-related stresses, deteriorating health and financial pressure.

Table 4: Difficulties in sleep-quality and-duration compared to time before the pandemic $(n=246)$

\begin{tabular}{lll}
\hline Score & Difficulty in Sleep Quality & Difficulty in Sleep Duration \\
\hline 0 & None & $\begin{array}{l}7-9 \mathrm{~h} \text { without interval change } \\
1\end{array}$ \\
$\begin{array}{l}\text { Suboptimal quality persisting without significant } \\
\text { change }\end{array}$ & $\begin{array}{l}<7 \mathrm{~h} \text { duration or }>9 \mathrm{~h} \text { persisting without } \\
\text { significant change } \\
\text { Add 1 point if suboptimal by } 1-2 \mathrm{~h} \text { increase }\end{array}$ \\
2 & $\begin{array}{l}\text { Suboptimal quality with significant change in } \\
\text { difficulty, lasted }<1 \text { month }\end{array}$ & $\begin{array}{l}\text { Duration further worsening by } 3-4 \mathrm{~h} \text { or } \\
\text { new such change since the pandemic began } \\
\text { that lasted }<1 \text { month }\end{array}$ \\
3 & $\begin{array}{l}\text { Suboptimal quality with significant change, lasting } \\
\text { Suboptimal duration with significant change, } \\
\text { lasting }>/=1 \text { month }\end{array}$
\end{tabular}

Note: Impaired sleep quality includes: difficulty in falling asleep, staying asleep, awakening in the middle of sleep with or without extremity pain, nightmares, early or difficulty awakening and unsatisfactory sleep.

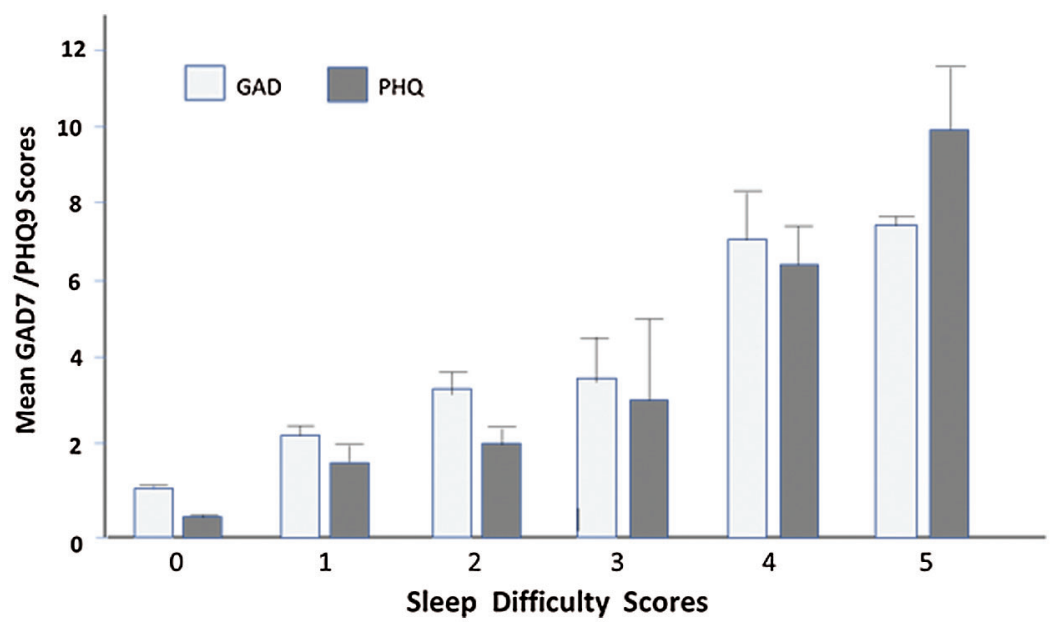

Figure 3: Sleep difficulty score with the mean GAD-7 and PHQ-9 symptom scores

\subsection{Financial Pressure and Job Dissatisfaction}

Overwhelming respondents expressed the need for the governmental support for the general public (87.8\%). The respondents anticipated their income decrease by less than $50 \%$ from the year before the pandemic were $39.8 \%$. Those who anticipated more than $50 \%$ of income decrease were $16.7 \%$.

Employment was reported in $2 / 3$ of the respondents. Among those with jobs, $22.7 \%$ reported dissatisfaction with their jobs. The dissatisfaction with one's job was shown as one of explanations for the depression. Getting a job or maintaining a job difficulty was also reported in $20.7 \%$ of the respondents. However, the amount of individualized income by itself had no significant association with the scores of anxiety, depression, TS/PTSD, or physical symptoms. 


\subsection{Hobby and Readiness/Resilience}

About $69 \%$ of the respondents reported difficulties carrying out their hobbies in their usual number of times per month during the pandemic. The mean anxiety score of the group not-participating a hobby was 2.9. However, the mean anxiety score of the group carrying out their hobbies 1-2 times a month or 34 or more times a month were lower at $2.0+/-0.3$ and $1.7+/-0.4$, respectively. Individuals' participation in their hobbies inversely correlated with the anxiety scores. The individuals who did not engaged in any hobby at all had the higher mean anxiety score $(p<0.01)$. Self-perceived resilience, one's capacity to bounce back from significant disturbance by stressors, had significant association inversely with the anxiety score $(r=-0.126, p<0.01)$. The dynamic relationships of the items in balancing act during pandemic are depicted in Fig. 4.

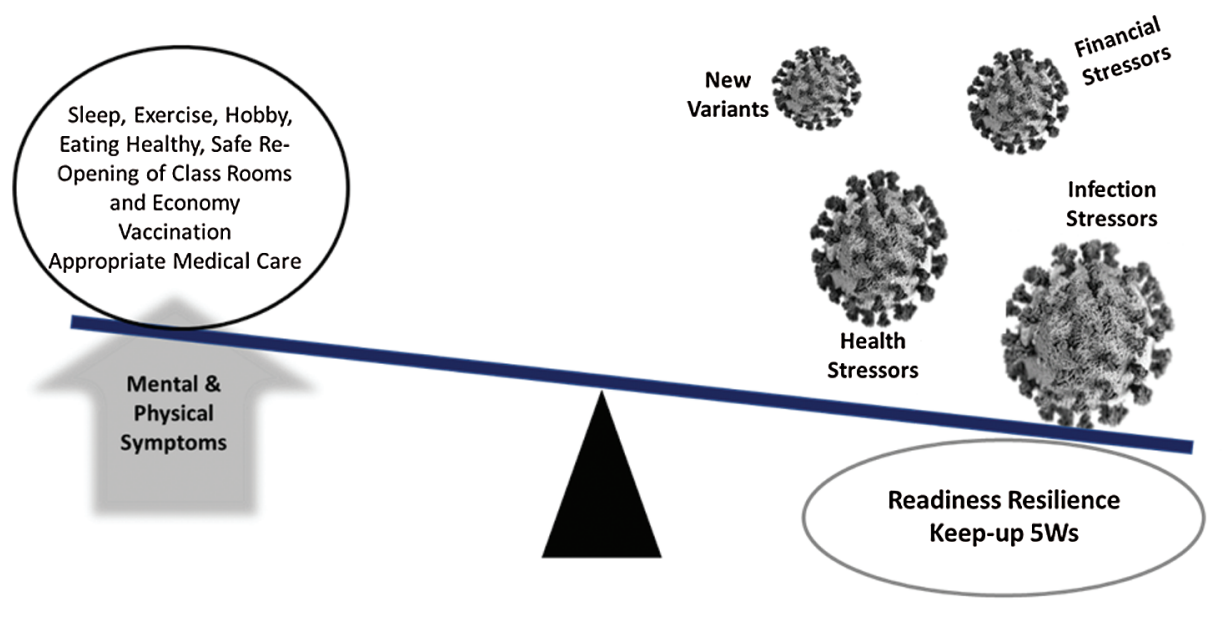

Balancing Act in Pandemic

Figure 4: Balancing Act in Pandemic. Items in circled areas counter-balance the pandemic stressors to maintain health. Appropriate public health measures are indicated for brevity as "5Ws: wear mask, wash hands, watch-out for physical distance, watch-out for crowded in-door space, while having minds close to others

\subsection{Helpfulness of the Survey}

The survey designed to assess the mental and physical symptoms of general public during the pandemic was found to be helpful in $82 \%$ of the respondents. Some reported it was helpful because the participation provided the chance to review and clearly identify their mental and physical changes that they were experiencing. Forty two percent of the respondents indicated their interests in participating follow-up or future research.

\section{Discussion}

In our study, there were significant number of individuals with the symptoms of generalized anxiety and major depression in South Korea, despite the successful management of the outbreaks. The degree of symptoms, however, were mostly in minimal and mild degrees. The individuals with more than or equal to mild degree of anxiety symptoms by GAD-7, depression symptoms by PHQ-9 scores were $16.2 \%$ and 9.3\%, respectively. The individuals with moderate degree of GAD-7 and PHQ-9 symptoms were 1.2\% and $2.4 \%$, respectively. No one was in higher degree. These percentages were apparently at the lower end of the published data by the systemic review based on the data collected in other countries [15]. However, direct interpretation of data among the published numbers is not possible becuases of the 
differences in study design and participants as well as different extent of COVID-19 status and socioeconomical situations in different countries. Different factors influenced our study results are following. Our study design of excluding the individuals with previous mental disorders, serious medical illnesses or in bereavement due to loss of a family [16,17] for all of which are associated with heighted stress responses influenced the result positively. For decreased mental health services and unclear access to therapies and medications during the COVID-19 crisis, accurate analysis of the individuals with mental illnesses could not be done.

Significant relationship and coexistence have been noted between the generalized anxiety, depression and multiple unexplained physical symptoms [18]. Especially, individuals with underlying mental illnesses also have a significantly increased risk for subsequently developing physical symptoms [19]. Unexplained multiple physcial symptoms could also represent somatization disorder or not-yet diagnosed, serious physical illness in clinically appropriate setting. For example, the scores of physical symptoms and sleep difficulty were found to correlate significantly with both depression and anxiety scores $(p<0.01)$ in our study. With the pandemic continuing, the minimal to mild degree of symptoms might have gotten worse or persisted for more than 6 months that could be diagnostic as having the GAD (DSM-5).

Home confinement has consequences [20]. Staying home has significant impacts on the family members. The highest percentage of the individuals with anxiety symptoms were noted in the group bringing-up young children. Mothers bringing-up a young child had the higher mean anxiety score compared to the fathers bringing-up a young child. The mean anxiety scores for the mothers raising children were significantly higher than the women in a marriage without child, 5.3 and 2.3, respectively. In South Korea, mothers are generally considered as the primary care-takers of young children. Three meals and snacks in-between, educating children, keeping them interested whole day and getting them to bed for young children, all fell more on the mothers to tend to as more the time spent at home [21]. The stress of getting infected and infecting others also increased as more of close contacts among the co-habitants were bound to happen around the clock. In fact, the contraction rate within the cohabitants of a house-hold turned out to be more than six times higher than the outside home in South Korea [2]. The parents bringing-up children also reported additional stress on their children's educational direction and future.

Depression or other psychiatric illness has been identified as individual risk factor for suicide [22]. Major depression is generally more prevalent than GAD, both in South Korea and USA. In 2013, South Korea was ranked the first among the OECD nations with the adolescents and young adults among the groups with the highest suicide rate [23]. Over sixteen percent of our respondents responding having had suicidal intent and close to $10 \%$ of these having tried should be addressed by appropriate evaluation and therapy. The prevalence of major depression in South Korea was $4.3 \%$ for life time and $1.7 \%$ for 12 months period before [24]. This prevalence of major depression was lower than that of the USA (6.8\% 12-month prevalence before the pandemic) although the fatality rate from suicide has been higher in South Korea. The reasons for the high suicide rate have been identified as financial and academic stressors which are both increased during pandemic. A range of symptom scores and prevalence of major depression had been reported based on different methods and make-up of the study [25,26]. The static glance of $2.4 \%$ rate by PHQ-9 likelihood in our study was higher than what was observed before, presumably due to the COVID-19 pandemic.

Depending on the type of stress and the individual's stress response skills or resilience, anxiety can result in positive effects as well as disease state. The fear of getting infected, infecting others and financial difficulties have been recognized as among the stressors of pandemic [27]. Many of our respondents found wearing masks, washing hands, and physical distancing as the reasons to maintain health rather than coerced, intrusive stressors. Majority of them reported their adherence most of time. Willingly participating in the public health measures were not only protecting from a massive spread of infection 
but it might have also meant the acceptance of the situation and solutions, at least temporarily. Being prepared and having regards for other might have also helped in being resilient. The host-resilience scores plus total number of stresses had a statistical significance with the generalized anxiety scores that might mean more resilience and less anxiety. Living with family members might also be a support and purpose for individuals to be resilient, shielding from isolation and loneliness and protecting from falling apart although stress of potentially infecting other family members being at the opposite side. The hostresilience may also shape how the individual perceives stimuli as stressors or challenges. It was reported $2 / 3$ will be resilient to the stresses of the pandemic [28], which mean $1 / 3$ may not.

The mental stress of the COVID pandemic has been exacerbated by the feeling of unknown, especially due to the mis-information from the social media [29,30]. The unpredictability of the COVID-19 epidemic progression may have resulted in significant psychological pressure on the populations that were vulnerable to the various stressors [31]. Furthermore, the misinformation from the media was also contributing to these mental stress [32-34]. Relatively short daily screen time spent on watching TV or other media by our study respondents, more than $73 \%$ reporting less than an hour, might have also played a part in demonstrating overall less degree of symptoms. In addition, most of respondents reported that they watched TV or other media together with family or friends which might also helped filtering the "fake news". Thus, more reliable and informative media and governmental reporting will be especially important when faced with the fear of unknown life during COVID-19 pandemic [35].

\section{Limitations and Future Prospective}

First limitation to the study is a potential sampling error due to the small number of respondents. In order to obtain the random samples of general public, we used a word-of-mouth approach instead of using a specific shopping program or social media. Despite there is representation from most of the regions of South Korea as listed in the demographic section, small number of participants limit the generalizability of data.

Second limitation might be the lack of RT-PCR or Serologic test results in all of our respondents. Their non-COVID-19 infection status was based on the individuals' responses. Only twelve individuals out of 246 reported actually having had SARS-Cov2 RT-PCR testing that were all negative. All 246 individuals reported no history of COVID-19 infection. Remoteness of asymptomatic infection was supported based on the results of the national investigations of antibodies to SARS-Cov- 2 that tested 4495 South Koreans randomly during the similar time period from $4 / 21 \sim 8 / 13 / 2020$ showing less than $0.05 \%$ positivity [2]. However, about $1 / 3$ of cases could be asymptomatic or subclinical infection speculated [2] and potential possibility of asymptomatic carriers are not entirely excluded.

Third limitation is a socio-cultural influence, potentially resulting in under-reporting of the mental symptoms. The internalized different stigma and its psychosocial correlates in Korean patients with mental illnesses have also been reported [36]. Mental illnesses are generally viewed as an "imperfection" to oneself and one's family in South Korea. For the physical symptoms reported by four in ten of our respondents, some physical symptoms might represent more culturally acceptable expressions of mental symptoms in the socio-cultural context. In previous years, the conservative 12 months prevalence rate of the generalized anxiety disorder in South Korea was 1\% [24]. Thus, our result of $1.2 \%$ static prevalence of generalized anxiety disorder based on GAD-7 likelihood is comparable to the published data. However, potential possibility of underreporting remains in this context.

Fourth limitation is selection bias through technological access by conducting on-line survey using smart phone or electronic mail. This prohibited those without access and resulted in mostly younger individuals responding. In South Korea, almost $1 / 2$ of individuals over 65 years-old live in relative poverty according to OECD data, reported in 2017. In addition to very young group, over 65 years-old group are at risk for developing mental illnesses and future research might be best conducted by a hybrid format additionally using traditional telephone. 
Fifth limitation is evolving nature of pandemic, limiting the scope and specific nature of culprits in its impacts. The results of present study based on evaluation of survey responses during the early phase of pandemic. Thus, further follow-up research is needed to see whether individuals' symptoms are persisting and what their responses to the post-vaccination era.

\section{Conclusion}

Despite the well-coordinated management of COVID-19 outbreaks in South Korea, our results show many individuals still had mental symptoms although mostly in minimal and mild degrees. In fact, many respondents reported their stresses in pandemic which had significant association with anxiety and depression symptoms. The physical symptoms and sleeping difficulties were correlated with higher degree of generalized anxiety and major depressive symptoms. This type of public health information survey during the pandemic will help the individuals and the health care professionals to identify and recognize these early signs for mental diseases. This early recognition will help the individuals to seek medical help to prevent or treat these mental symptoms before they become more serious mental crisis.

Acknowledgement: We are thankful for our respondents for their time and participation in the survey.

Funding Statement: The authors received no specific funding for this study.

Conflicts of Interest: The authors declare that they have no conflicts of interest to report regarding the present study.

\section{References}

1. World Health Organization (WHO) (2020). WHO Coronavirus Disease (COVID-19) Dashboard. https://covid19. who.int/.

2. Korean Center for Disease Control (KCDC) (2021). The updates on COVID-19 in Korea as of Feb. 25, 2021. https://www.cdc.go.kr/board/board.es?mid = a30402000000\&bid=0030.

3. Dighe, A., Cattarino, L., Cuomo-Dannenburg, G., Skarp, J., Imai, N. et al. (2020). Response to COVID-19 in South Korea and implications for lifting stringent interventions. BMC Medicine, 18(1), 321. DOI 10.1186/ s12916-020-01791-8.

4. Song, J. Y., Yun, J. G., Noh, J. Y., Cheong, H. J., Kim, W. J. (2020). COVID-19 in South Korea-challenges of subclinical manifestations. New England Journal of Medicine, 382(19), 1858-1859. DOI 10.1056/NEJMc2001801.

5. Johansson, M. A., Quandelacy, T. M., Kada, S., Prasad, P. V., Steele, M. et al. (2021). SARS-CoV-2 transmission from people without COVID-19 symptoms. Journal of the American Medical Association Network Open, 4(1), e2035057. DOI 10.1001/jamanetworkopen.2020.35057.

6. Sprang, G., Silman, M. (2013). Posttraumatic stress disorder in parents and youth after health-related disasters. Disaster Medicine and Public Health Preparedness, 7(1), 105-110. DOI 10.1017/dmp.2013.22.

7. Czeisler, M. E., Marynak, K., Clarke, K. E. N., Salah, Z., Shakya, I. et al. (2020). Delay or avoidance of medical care because of COVID-19-related concerns-United States. Morbidity and Mortality Weekly Report, 69(36), 12501257. DOI 10.15585/mmwr.mm6936a4 2020-06.

8. Baldi, E., Sechi, G. M., Mare, C., Canevari, F., Brancaglione, A. et al. (2020). Out-of-Hospital Cardiac Arrest during the COVID-19 Outbreak in Italy. New England Journal of Medicine, 383(5), 496-498. DOI 10.1056/ NEJMc2010418.

9. Woolf, S. H., Chapman, D. A., Sabo, R. T., Weinberger, D. M., Hill, L. et al. (2020). Excess deaths from COVID-19 and other causes, March-July 2020. Journal of the American Medical Association, 324(15), 15621564. DOI 10.1001/jama.2020.19545.

10. National Center for Health Statistics (NCHS) (2020). Early release of selected mental health estimates based on data from the January-June 2019 national health interview survey. www.cdc.gov/nchs/data/nhis/ earlyrelease/ ERmentalhealth-508.pdf. 
11. Kroenke, K., Spitzer, R. L., Williams, J. B. (2001). The PHQ-9: Validity of a brief depression severity measure. Journal of General Internal Medicine, 16(9), 606-613. DOI 10.1046/j.1525-1497.2001.016009606.x.

12. Spitzer, R. L., Kroenke, K., Williams, J. B., Lowe, B. (2006). A brief measure for assessing generalized anxiety disorder: The GAD-7. Archives of Internal Medicine, 166(10), 1092-1097. DOI 10.1001/archinte.166.10.1092.

13. Morin, C. M., Belleville, G., Belanger, L., Ivers, H. (2011). The Insomnia Severity Index: Psychometric indicators to detect insomnia cases and evaluate treatment response. Sleep, 34(5), 601-608. DOI 10.1093/sleep/34.5.601.

14. Okun, M. L., Kravitz, H. M., Sowers, M. F., Moul, D. E., Buysse, D. J. (2009). Psychometric evaluation of the Insomnia Symptom Questionnaire: A self-report measure to identify chronic insomnia. Journal of Clinical Sleep Medicine, 5(1), 41-51.

15. Xiong, J., Lipsitz, O., Nasri, F., Lui, L. M. W. (2020). Impact of COVID-19 pandemic on mental health in the general population: A systematic review. Journal of Affective Disorders, 277, 55-64. DOI 10.1016/j. jad.2020.08.001.

16. Zisook, S., Corruble, E., Duan, N., Iglewicz, A., Karam, E. G. et al. (2012). The bereavement exclusion and DSM-5. Depression and Anxiety, 29(5), 425-443. DOI 10.1002/da.21927.

17. Seiler, A., von Kenel, R., Slavich, G. M. (2020). The psychobiology of bereavement and health: A conceptual review from the perspective of social signal transduction theory of depression. Review Frontiers in Psychiatry, 11, 1-16. DOI 10.3389 fpsyz565239.

18. Komaroff, A. L. (2005). Harvard medical school family health guide. New York, NY, USA: Free Press.

19. Momen, N. C., Plana-Ripoll, O., Agerbo, E., Benros, M. E., Borglum, A. D. et al. (2020). Association between mental disorders and subsequent medical conditions. New England Journal of Medicine, 382(18), 1721-1731. DOI 10.1056/NEJMoa1915784.

20. de Pietri, S., Chiorri, C. (2021). Early impact of COVID quarantine on the perceived changes of anxiety symptoms in a non-clinical non-infected Italian sample effect of COVID-19 quarantine on anxiety. Journal of Affective Disorders Reports, 4, 100078.

21. Choi, A. R. (2020). Issues involving corona 19 and child-caring. Journal of Humanities and Social Science, 21, 11(4), 1379-1389.

22. Fazel, S., Runeson, B. (2020). Suicide. New England Journal of Medicine, 382(3), 266-274. DOI 10.1056/ NEJMra1902944.

23. Organisation for Economic Co-operation and Development (OECD) (2014). Focus on health. Making Mental Health Count. https://www.oecd.org/els/health-systems/Focus-on-Health-Making-Mental-Health-Count.pdf.

24. Cho, M. J., Kim, J. K., Jeon, H. J., Suh, T., Chung, I. W. et al. (2007). Lifetime and 12-month prevalence of DSMIV psychiatric disorders among Korean adults. Journal of Nervous and Mental Disease, 195(3), 203-210. DOI 10.1097/01.nmd.0000243826.40732.45.

25. Koo, S. K. (2018). Depression status in Korea. Osong Public Health and Research Perspectives, 9(4), 141-142. DOI 10.24171/j.phrp.2018.9.4.01.

26. Shin, C., Kim, Y., Park, S., Yoon, S., Ko, Y. H. et al. (2017). Prevalence and associated factors of depression in general population of Korea: Results from the Korea National Health and Nutrition Examination Survey, 2014. Journal of Korean Medical Science, 32(11), 1861-1869. DOI 10.3346/jkms.2017.32.11.1861.

27. Taylor, S., Landry, C. A., Paluszek, M. M., Fergus, T. A., McKay, D. (2020). Development and initial validation of the COVID Stress Scales. Journal of Anxiety Disorders, 72, 102232. DOI 10.1016/j.janxdis.2020.102232.

28. Pfefferbaum, B., North, C. S. (2020). Mental health and the COVID-19 pandemic. New England Journal of Medicine, 383(6), 510-512. DOI 10.1056/NEJMp2008017.

29. Lăzăroiu, G., Horak, J., Valaskova, K. (2020). Scaring ourselves to death in the time of COVID-19: Pandemic awareness, virus anxiety, and contagious fear. Linguistic and Philosophical Investigations, 19, 114-120. DOI 10.22381/LPI1920208.

30. Lăzăroiu, G., Adams, C. (2020). Viral panic and contagious fear in scary times: The proliferation of COVID-19 misinformation and fake news. Analysis and Metaphysics, 19, 80-86. DOI 10.22381/AM1920209. 
31. Pera, A. (2020). Depressive symptoms, anxiety disorder, and suicide risk during the COVID-19 pandemic. Frontiers in Psychology, 11, 572699. DOI 10.3389/fpsyg.2020.572699.

32. Bratu, S. (2020). The fake news sociology of COVID-19 pandemic fear: Dangerously inaccurate beliefs, emotional contagion, and conspiracy ideation. Linguistic and Philosophical Investigations, 19, 128-134. DOI 10.22381/ LPI19202010.

33. Clark, A. (2020). COVID-19-related misinformation: Fabricated and unverified content on social media. Analysis and Metaphysics, 19, 87-93. DOI 10.22381/AM19202010.

34. Dobson-Lohman, E., Potcovaru, A. M. (2020). Fake news content shaping the COVID-19 pandemic fear: Virus anxiety, emotional contagion, and responsible media reporting. Analysis and Metaphysics, 19, 94-100. DOI 10.22381/AM19202011.

35. Popescu Ljungholm, D., Olah, M. L. (2020). Regulating fake news content during COVID-19 pandemic: Evidence-based reality, trustworthy sources, and responsible media reporting. Review of Contemporary Philosophy, 19, 43-49. DOI 10.22381/RCP1920203.

36. Kim, W. J., Song, Y. J., Ryu, H. S., Ryu, V., Kim, J. M. et al. (2015). Internalized stigma and its psychosocial correlates in Korean patients with serious mental illness. Psychiatry Research, 225(3), 433-439. DOI 10.1016/ j.psychres.2014.11.071. 